\title{
FINITE ELEMENT APPROXIMATION OF A PARABOLIC INTEGRO-DIFFERENTIAL EQUATION WITH A WEAKLY SINGULAR KERNEL
}

\author{
C. CHEN, V. THOMÉE, AND L. B. WAHLBIN
}

\begin{abstract}
We give error estimates for the numerical solution by means of the Galerkin finite element method of an integro-differential equation of parabolic type with a memory term containing a weakly singular kernel. Optimal-order estimates are shown for spatially semidiscrete and completely discrete methods. Special attention is paid to the regularity of the exact solution.
\end{abstract}

\section{INTRODUCTION}

We shall consider the initial value problem (with $u_{t}=\partial u / \partial t$ )

$$
\begin{aligned}
u_{t}+A u & =\int_{0}^{t} K(t-s) B u(s) d s+f(t) \text { in } \Omega, \text { for } t>0, \\
u & =0 \text { on } \partial \Omega, t>0 \\
u(0) & =u_{0} \text { in } \Omega,
\end{aligned}
$$

where $A$ is a linear positive selfadjoint elliptic and $B$ a general partial differential operator of second order with smooth, time-independent coefficients, where $K$ is a weakly singular kernel $K(t)$ such that

$$
|K(t)| \leq C t^{-\alpha} \text { with } 0 \leq \alpha<1 \text {, for } t>0,
$$

and where $\Omega$ is a sufficiently smooth domain in $R^{d}, d \geq 1$. Integro-differential equations of this nature appear in applications such as heat conduction in materials with memory, population dynamics, and visco-elasticity; cf., e.g., Friedman and Shinbrot [3], Heard [5], and Renardy, Hrusa, and Nohel [12]. For equations with nonsmooth kernels such as in (1.2), we refer to Grimmer and Pritchard [4], Lunardi and Sinestrari [10], and Lorenzi and Sinestrari [9] and references therein. Finite element methods for problems of the form (1.1) with a smooth kernel $K$ have been discussed in, e.g., Sloan and Thomée [13], Yanik and Fairweather [15], Thomée and Zhang [14], LeRoux and Thomée [6], Cannon and Lin [1], and Lin, Thomée, and Wahlbin [7].

Received June 21, 1990.

1991 Mathematics Subject Classification. Primary 65M60, 65R20.

Key words and phrases. Parabolic, integro-differential equation, memory, Galerkin, finite element, weakly singular kernel.

The second author was supported by the National Science Foundation, USA.

The third author was supported by the Army Research Office through the Mathematical Sciences Institute, Cornell, and by the National Science Foundation, USA. 
For the numerical solution we assume that we are given a family $\left\{S_{h}\right\}$ of finite-dimensional subspaces of $H_{0}^{1}=H_{0}^{1}(\Omega)$ such that

$$
\inf _{\chi \in S_{h}}\left\{\|v-\chi\|+h\|v-\chi\|_{1}\right\} \leq C h^{2}\|v\|_{2}, \quad \forall v \in H^{2} \cap H_{0}^{1},
$$

where $\|\cdot\|$ is the norm in $L_{2}=L_{2}(\Omega)$ and $\|\cdot\|_{s}$ that in $H^{s}=H^{s}(\Omega)$.

We consider first the semidiscrete problem of finding $u_{h}:[0, \infty) \rightarrow S_{h}$ such that

$$
\begin{aligned}
\left(u_{h, t}, \chi\right)+A\left(u_{h}, \chi\right) & =\int_{0}^{t} K(t-s) B\left(u_{h}(s), \chi\right) d s+(f(t), \chi), \\
u_{h}(0) & =u_{0 h}, \quad \forall \chi \in S_{h}, t>0,
\end{aligned}
$$

where $(\cdot, \cdot)$ is the inner product in $L_{2}$ and $A(\cdot, \cdot)$ and $B(\cdot, \cdot)$ are the bilinear forms on $H_{0}^{1}$ associated with the differential operators $A$ and $B$, and where $u_{0 h}$ is an appropriate approximation in $S_{h}$ of the initial data in (1.1). We shall show that, for each $T>0$, we then have the error estimate

$$
\left\|u_{h}(t)-u(t)\right\| \leq C_{T} h^{2}\left\{\left\|u_{0}\right\|_{2}+\int_{0}^{t}\left\|u_{t}\right\|_{2} d s\right\} \quad \text { for } t \leq T .
$$

We shall also consider the discretization in time of (1.4). Thus, let $k$ be a time step, and let $U^{n} \in S_{h}$ be the approximation of the exact solution of (1.1) at time $t_{n}=n k$. The time discretization considered will be based on the backward difference quotient $\bar{\partial}_{t} U^{n}=\left(U^{n}-U^{n-1}\right) / k$. The integral term then has to be evaluated by numerical quadrature from the values of the $U^{n}$, but since the integrand is singular, even when the solution is smooth, we shall use product integration: We shall approximate $\phi$ in $J_{n}(\phi)=\int_{0}^{t_{n}} K\left(t_{n}-s\right) \phi(s) d s$ by the piecewise constant function taking the value $\phi\left(t_{j}\right)$ in $\left(t_{j}, t_{j+1}\right)$, and thus use

where

$$
J_{n}(\phi) \approx Q_{n}(\phi)=\sum_{j=0}^{n-1} \int_{t_{j}}^{t_{j+1}} K\left(t_{n}-s\right) \phi\left(t_{j}\right) d s=\sum_{j=0}^{n-1} \kappa_{n-j} \phi\left(t_{j}\right),
$$

$$
\kappa_{j}=\int_{t_{j-1}}^{t_{j}} K(s) d s .
$$

Our completely discrete scheme is therefore

$$
\begin{aligned}
& \left(\bar{\partial}_{t} U^{n}, \chi\right)+A\left(U^{n}, \chi\right) \\
& \quad=\sum_{j=0}^{n-1} \kappa_{n-j} B\left(U^{j}, \chi\right)+\left(f\left(t_{n}\right), \chi\right), \quad \forall \chi \in S_{h}, n \geq 1, \\
& U^{0}=u_{0 h} .
\end{aligned}
$$

For this completely discrete method we shall show

$$
\left\|U^{n}-u\left(t_{n}\right)\right\| \leq C_{T}\left(h^{2}+k\right)\left\{\left\|u_{0}\right\|_{2}+\int_{0}^{t_{n}}\left(\left\|u_{t t}\right\|+\left\|u_{t}\right\|_{2}\right) d s\right\} \quad \text { for } t \leq T
$$

Before we analyze these discrete methods, we shall discuss the existence and regularity of the solution of (1.1) and show, in particular, that the regularity 
required for the estimates (1.5) and (1.8) are satisfied under appropriate assumptions on the data. In the case of a weakly singular kernel the regularity of the solution with respect to time is limited, which makes higher-order quadrature formulas less attractive, as well as quadratures based on the use of sparser sets of time levels, such as those treated in [13] and [6].

\section{AN EXISTENCE AND REGULARITY RESULT}

In this section we shall study the existence and regularity of the solutions of (1.1) and show, in particular, that the regularity required for the error estimates (1.5) and (1.8) holds under appropriate assumptions on the data of (1.1).

We shall need the following version of Gronwall's lemma.

Lemma 1. Assume that $y$ is a nonnegative function in $L_{1}(0, T)$ which satisfies

$$
y(t) \leq b(t)+\beta \int_{0}^{t}(t-s)^{-\alpha} y(s) d s \quad \text { for } 0<t \leq T,
$$

where $b(t) \geq 0, \beta \geq 0$. Then there is a constant $C_{T}$ such that

$$
y(t) \leq b(t)+C_{T} \int_{0}^{t}(t-s)^{-\alpha} b(s) d s \quad \text { for } t \leq T .
$$

Proof. Let $K_{1}(s)=\beta s^{-\alpha}$ for $0<s<T$, and let $K_{1} * f$ denote the convolution

$$
\left(K_{1} * f\right)(t)=\int_{0}^{t} K_{1}(t-s) f(s) d s .
$$

Recall that this is a bounded operator on $L_{1}(0, T)$. With $K_{i}$ the kernel of the $i$ times iterated convolution, we have

$$
K_{i}(s) \leq C(i, \alpha) s^{i(1-\alpha)-1},
$$

and we easily see that $K_{i} * b(t) \leq C K_{1} * b(t)$ for $i \geq 2$. Hence, applying $K_{1} *$ to (2.1) $i$ times in succession, we obtain

$$
y(t) \leq b(t)+C\left(K_{1} * b\right)(t)+\left(K_{i} * y\right)(t) .
$$

For $i(1-\alpha)-1>0$, we have

$$
\left(K_{i} * y\right)(t) \leq C \int_{0}^{t} y(s) d s
$$

and we can use the ordinary Gronwall lemma. Since

$$
\int_{0}^{t} b(s) d s \leq C\left(K_{1} * b\right)(t),
$$

this concludes the proof.

We shall also need the following lemma.

Lemma 2. Let $K \in L_{1}(0, T)$. Then for each $\varepsilon>0$ there is a constant $C_{\varepsilon}=$ $C_{\varepsilon}\left(\|K\|_{L_{1}(0, T)}\right)$ such that

$$
\begin{aligned}
& \mid \int_{0}^{T} \int_{0}^{t} K(t-s) f(s) f(t) d s d t \mid \\
& \leq \varepsilon \int_{0}^{T} f(t)^{2} d t+C_{\varepsilon} \int_{0}^{T}|K(T-t)| \int_{0}^{t} f(s)^{2} d s d t
\end{aligned}
$$


Proof. In this proof, let $(\cdot, \cdot)$ and $\|\cdot\|$ denote the inner product and norm in $L_{2}(0, T)$. We have, using the Cauchy-Schwarz inequality,

$$
\begin{aligned}
|(K * f)(t)|^{2} & \leq\left(\int_{0}^{t}|K(s)|^{1 / 2}|K(s)|^{1 / 2}|f(t-s)| d s\right)^{2} \\
& \leq\|K\|_{L_{1}(0, T)} \int_{0}^{t}|K(s)| f^{2}(t-s) d s
\end{aligned}
$$

Hence, integrating with respect to $t$ and changing the order of integration, and then changing variables,

$$
\begin{aligned}
\|K * f\|^{2} & \leq\|K\|_{L_{1}(0, T)} \int_{0}^{T}|K(s)| \int_{s}^{T} f^{2}(t-s) d t d s \\
& =\|K\|_{L_{1}(0, T)} \int_{0}^{T}|K(T-\tau)| \int_{0}^{\tau} f^{2}(\sigma) d \sigma d \tau .
\end{aligned}
$$

Hence, for the left-hand side of (2.2),

$$
\begin{aligned}
|(K * f, f)| & \leq\|K * f\|\|f\| \leq \varepsilon\|f\|^{2}+\frac{1}{4 \varepsilon}\|K * f\|^{2} \\
& \leq \varepsilon\|f\|^{2}+\frac{1}{4 \varepsilon}\|K\|_{L_{1}(0, T)} \int_{0}^{T}|K(T-t)| \int_{0}^{t} f(s)^{2} d s d t
\end{aligned}
$$

which is the desired inequality.

The following is our main existence and regularity result.

Theorem 1. Assume that $u_{0} \in H^{\beta} \cap H_{0}^{1}, f \in \mathscr{C}\left([0, T] ; H^{\beta-2}\right)$ and $t^{\gamma} f_{t} \in$ $L_{\infty}\left(0, T ; H^{\beta-2}\right)$ with $\beta>2,0<\gamma<1$. Then there exists a unique solution of $(1.1)$ in $\mathscr{C}\left([0, T] ; L_{2}\right)$. Furthermore, $u \in \mathscr{C}\left([0, T] ; H^{2} \cap H_{0}^{1}\right), u_{t} \in$ $\mathscr{C}\left([0, T] ; L_{2}\right) \cap L_{1}\left(0, T ; H^{2} \cap H_{0}^{1}\right)$, and $u_{t t} \in L_{1}\left(0, T ; L_{2}\right)$.

Proof. We shall use the procedure of Faedo-Galerkin. Let $\left\{\phi_{j}\right\}_{1}^{\infty}$ be the eigenfunctions of $A$. We first seek $u^{n} \in \mathscr{S}_{n}=\operatorname{span}\left[\phi_{1}, \ldots, \phi_{n}\right]$ satisfying

$$
\begin{aligned}
u_{t}^{n}+A u^{n} & =\int_{0}^{t} K(t-s) P_{n} B u^{n}(s) d s+P_{n} f(t) \quad \text { in } \Omega, \text { for } t \geq 0, \\
u^{n} & =0 \text { on } \partial \Omega, \quad t \geq 0 \\
u^{n}(0) & =P_{n} u_{0} \quad \text { in } \Omega .
\end{aligned}
$$

Here, $P_{n}$ denotes the $L_{2}$ projection into $\mathscr{S}_{n}$. By standard arguments, cf., e.g., Linz [8], this system of ordinary integro-differential equations has a solution $u^{n} \in \mathscr{C}^{1}([0, T]) \cap \mathscr{C}^{2}((0, T])$.

We shall next derive a priori estimates for $u^{n}$. We first show that, independently of $n$,

$$
\begin{array}{r}
\left(\int_{0}^{T}\left\|u_{t}^{n}\right\|_{2}^{p} d t\right)^{1 / p}+\left(\int_{0}^{T}\left\|u_{t t}^{n}\right\|^{p} d t\right)^{1 / p} \leq C_{T} M_{\beta, \gamma} \\
\text { for some } p=p(\alpha)>1
\end{array}
$$


where

$$
M_{\beta, \gamma}=M_{\beta, \gamma}\left(u_{0}, f\right) \equiv\left\|u_{0}\right\|_{\beta}+\|f(0)\|_{\beta-2}+\sup _{s \leq t}\left(s^{\gamma}\left\|f_{t}(s)\right\|_{\beta-2}\right)
$$

Differentiating (2.3), we find that $v^{n}=u_{t}^{n}$ satisfies

$$
\begin{aligned}
v_{t}^{n}+A v^{n}= & K(t) P_{n} B P_{n} u_{0} \\
& +\int_{0}^{t} K(t-s) P_{n} B v^{n}(s) d s+P_{n} f_{t}(t) \quad \text { in } \Omega, t>0, \\
v^{n}= & 0 \text { on } \partial \Omega, \quad t>0, \\
v^{n}(0)= & -A P_{n} u_{0}+P_{n} f(0) \quad \text { in } \Omega .
\end{aligned}
$$

We now define $w^{j}=w^{n, j}, j \geq 1$, inductively by

$$
\begin{aligned}
w_{t}^{1}+A w^{1} & =K(t) P_{n} B P_{n} u_{0}+P_{n} f_{t}(t) \quad \text { in } \Omega, t>0 \\
w^{1} & =0, \quad \text { on } \partial \Omega, t>0, \\
w^{1}(0) & =-A P_{n} u_{0}+P_{n} f(0),
\end{aligned}
$$

and then, for $j \geq 2$,

$$
\begin{aligned}
w_{t}^{j}+A w^{j} & =\int_{0}^{t} K(t-s) P_{n} B w^{j-1}(s) d s \equiv W^{j-1}(t) \quad \text { in } \Omega, t>0, \\
w^{j} & =0 \text { on } \partial \Omega, t>0 \\
w^{j}(0) & =0 \text { in } \Omega .
\end{aligned}
$$

Setting $z^{j}=v^{n}-\sum_{l=1}^{j} w^{l}$, we find for $j \geq 1$

$$
\begin{aligned}
z_{t}^{j}+A z^{j} & =\int_{0}^{t} K(t-s) P_{n} B\left(z^{j}+w^{j}\right)(s) d s \\
& \equiv \int_{0}^{t} K(t-s) P_{n} B z^{j}(s) d s+g^{j} \quad \text { in } \Omega, t>0, \\
z^{j} & =0 \quad \text { on } \partial \Omega, t>0, \\
z^{j}(0) & =0 \quad \text { in } \Omega .
\end{aligned}
$$

We shall show below that, for any $j \geq 1$ and $\delta$ with $2<\delta<\beta$, there is a constant $C_{j}=C_{j}(\alpha, \delta)$ such that

$$
\left\|w^{j}(t)\right\|_{\delta} \leq C_{j} t^{-1+(\beta-\delta) / 2+(j-1)(1-\alpha)} M_{\beta, \gamma} .
$$

Assuming this for a moment, we conclude first that

$$
\left(\int_{0}^{T}\left\|w^{j}\right\|_{2}^{p} d t\right)^{1 / p} \leq C_{j, T} M_{\beta, \gamma} \text { for some } p>1, j \geq 1
$$


In order to bound $z^{j}$, we first note that by (2.7)

$$
\begin{aligned}
\left\|g^{j}\right\| & =\left\|\int_{0}^{t} K(t-s) P_{n} B w^{j}(s) d s\right\| \\
& \leq C \int_{0}^{t}(t-s)^{-\alpha} s^{-1+(j-1)(1-\alpha)} d s M_{\beta, \gamma} \leq C M_{\beta, \gamma} \quad \text { if } \quad j(1-\alpha) \geq 1 .
\end{aligned}
$$

We now multiply (2.6) by $2 A z^{j}(t)$ and integrate to obtain

$$
\begin{aligned}
& \left\|A^{1 / 2} z^{j}(T)\right\|^{2}+2 \int_{0}^{T}\left\|A z^{j}\right\|^{2} d t \\
& \quad \leq C \int_{0}^{T} \int_{0}^{t}(t-s)^{-\alpha}\left\|z^{j}(s)\right\|_{2}\left\|z^{j}(t)\right\|_{2} d s d t+C \int_{0}^{T}\left\|z^{j}\right\|_{2} d s M_{\beta, \gamma} .
\end{aligned}
$$

Hence, using Lemma 2 with $\varepsilon$ suitably chosen for the double integral, and the Cauchy-Schwarz inequality for the last term, we have

$$
2 \int_{0}^{T}\left\|z^{j}\right\|_{2}^{2} d t \leq C M_{\beta, \gamma}^{2}+\int_{0}^{T}\left\|z^{j}\right\|_{2}^{2} d t+C \int_{0}^{T}(T-t)^{-\alpha} \int_{0}^{t}\left\|z^{j}(s)\right\|_{2}^{2} d s d t .
$$

Moving the second term on the right over to the left and using Lemma 1, we conclude that

$$
\int_{0}^{T}\left\|z^{j}\right\|_{2}^{2} d t \leq C_{T} M_{\beta, \gamma}^{2} \text { for } j(1-\alpha) \geq 1 .
$$

In particular, the estimate for $u_{t}^{n}$ in (2.4) follows from this and (2.8).

It remains to show (2.7). For this purpose we first recall that the semigroup $E(t)$ generated by $-A$ satisfies, for $\phi \in H^{\mu}$ with $\phi=0$ on $\partial \Omega$ if $\mu \geq \frac{1}{2}$,

$$
\|E(t) \phi\|_{\nu} \leq C t^{-(\nu-\mu) / 2}\|\phi\|_{\mu}, \quad 0 \leq \mu \leq \nu, \quad \mu<2.5 .
$$

(For $\mu \geq 2.5$, further boundary conditions have to be imposed on $\phi$.) We have the representation

$$
\begin{aligned}
w^{1}= & -E(t) A P_{n} u_{0}+E(t) P_{n} f(0)+\int_{0}^{t} E(t-s) K(s) P_{n} B u_{0} d s \\
& +\int_{0}^{t} E(t-s) P_{n} f_{t}(s) d s,
\end{aligned}
$$

so that for $\delta<\beta$ (which we may clearly assume less than 2.5),

$$
\begin{aligned}
\left\|w^{1}\right\|_{\delta} \leq & C t^{-1+(\beta-\delta) / 2}\left(\left\|u_{0}\right\|_{\beta}+\|f(0)\|_{\beta-2}\right) \\
& +C \int_{0}^{t}(t-s)^{-1+(\beta-\delta) / 2} s^{-\alpha} d s\left\|u_{0}\right\|_{\beta} \\
& +C \int_{0}^{t}(t-s)^{-1+(\beta-\delta) / 2} s^{-\gamma}\left(s^{\gamma}\left\|f_{t}(s)\right\|_{\beta-2}\right) d s \\
\leq & C t^{-1+(\beta-\delta) / 2} M_{\beta, \gamma} .
\end{aligned}
$$

We now proceed with a proof of (2.7) by induction for $j \geq 2$ and assume the 
result holds for $j-1$. We note that then, for $2<\delta<\beta$,

$$
\begin{aligned}
\left\|W^{j-1}(t)\right\|_{\delta-2} & \leq C \int_{0}^{t}(t-s)^{-\alpha}\left\|w^{j-1}(s)\right\|_{\delta} d s \\
& \leq C_{j-1} \int_{0}^{t}(t-s)^{-\alpha} s^{-1+(\beta-\delta) / 2+(j-2)(1-\alpha)} d s M_{\beta, \gamma} \\
& \leq C_{j-1} t^{-1+(\beta-\delta) / 2+(j-1)(1-\alpha)} M_{\beta, \gamma} .
\end{aligned}
$$

Thus, by (2.9), if $\varepsilon<\delta<\beta$, we obtain

$$
\begin{aligned}
\left\|w^{j}(t)\right\|_{\varepsilon} & =\left\|\int_{0}^{t} E(t-s) W^{j-1}(s) d s\right\|_{\varepsilon} \\
& \leq C_{j-1} \int_{0}^{t}(t-s)^{-1+(\delta-\varepsilon) / 2} s^{-1+(\beta-\delta) / 2+(j-1)(1-\alpha)} d s M_{\beta, \gamma} \\
& \leq C_{j-1} t^{-1+(\beta-\varepsilon) / 2+(j-1)(1-\alpha)} M_{\beta, \gamma},
\end{aligned}
$$

which completes the proof of (2.7), and thus of the estimate for the first term in (2.4). Clearly, we then also have $\left\|u^{n}(t)\right\|_{2} \leq C$, and it follows easily from (2.5) that the bound for $u_{t t}^{n}$ in (2.4) is satisfied, and hence also that

$$
\left\|u^{n}(t)\right\|_{2}+\left\|u_{t}^{n}(t)\right\| \leq C \text { for } 0 \leq t \leq T .
$$

We next proceed with a limiting argument. Writing (1.1) in weak form, we have

$$
\begin{aligned}
\left(u_{t}^{n}, \phi_{m}\right)+A\left(u^{n}, \phi_{m}\right) & =\int_{0}^{t} K(t-s) B\left(u^{n}, \phi_{m}\right) d s+\left(f(t), \phi_{m}\right) \text { for } m \leq n, \\
u^{n}(0) & =P_{n} u_{0} .
\end{aligned}
$$

By (2.10), a subsequence $u^{n}$ converges weak* in $L_{\infty}\left(0, T ; H^{2}\right)$, and we refer to that limit as $u$. By (2.4) we may also assume that a (further) subsequence $u_{t}^{n}$ converges weakly in $L_{p}\left(0, T ; H^{2}\right)$. Since $u_{t}^{n}$ converges to $u_{t}$ in the distribution sense, the weak limit is $u_{t}$ also in $L_{p}\left(0, T ; H^{2}\right)$. In particular, $u_{t} \in L_{p}\left(0, T ; H^{2}\right)$. Similarly, by $(2.4)$ again, $u_{t t}^{n} \rightarrow u_{t t}$ in $L_{p}\left(0, T ; L_{2}\right)$. By (2.10) we may further assume that $\left(u_{t}^{n}, \phi_{m}\right), A\left(u^{n}, \phi_{m}\right)$, and $B\left(u^{n}, \phi_{m}\right)$ all converge weak $*$ in $L_{\infty}(0, T)$, and the limits are $\left(u_{t}, \phi_{m}\right), A\left(u, \phi_{m}\right)$, and $B\left(u, \phi_{m}\right)$, respectively. Hence, for any $\psi \in L_{1}(0, T)$ and $m>0$,

$$
\begin{aligned}
\int_{0}^{T}[ & \left(u_{t}(t), \phi_{m}\right)+A\left(u(t), \phi_{m}\right) \\
& \left.\quad-\int_{0}^{t} K(t-s) B\left(u(s), \phi_{m}\right) d s-\left(f(t), \phi_{m}\right)\right] \psi(t) d t=0 .
\end{aligned}
$$

Since $\left(u_{t}, \phi_{m}\right)$ and $\left(u_{t t}, \phi_{m}\right)$ both belong to $L_{1}(0, T)$, we have that $\left(u_{t}, \phi_{m}\right)$ is actually continuous on $[0, T]$. One similarly sees that $A\left(u, \phi_{m}\right)$ and $B\left(u, \phi_{m}\right)$ are continuous. Hence, using the density of the $\phi_{m}$, one obtains the weak form of (1.1). Since $u \in L_{1}\left(0, T ; H^{2} \cap H_{0}^{1}\right)$ and $u_{t} \in L_{1}\left(0, T ; H^{2} \cap H_{0}^{1}\right)$, we have actually $u \in \mathscr{C}\left([0, T] ; H^{2} \cap H_{0}^{1}\right)$. Similarly, $u_{t} \in \mathscr{C}\left([0, T] ; L_{2}\right)$, and one concludes that $(1.1)$ holds as an equation in $\mathscr{C}\left([0, T] ; L_{2}\right)$.

This completes the proof of the theorem. 
To see that, in general, $u_{t t}$ blows up as $t \rightarrow 0$, consider the problem

$$
\begin{aligned}
u_{t}+A u & =\int_{0}^{t}(t-s)^{-\alpha} A u(s) d s \text { in } \Omega, \text { for } t>0, \\
u & =0 \text { on } \partial \Omega, \text { for } t>0, \\
u(0) & =\phi \quad \text { in } \Omega,
\end{aligned}
$$

where $\phi$ is an eigenfunction of $A$ corresponding to the eigenvalue $\lambda$. Setting $u(x, t)=\phi(x) y(t)$, we have for the scalar function $y$

$$
\begin{aligned}
y^{\prime}+\lambda y & =\lambda \int_{0}^{t}(t-s)^{-\alpha} y(s) d s \text { for } t>0, \\
y(0) & =1,
\end{aligned}
$$

and hence

$$
y^{\prime \prime}(t)=\lambda t^{-\alpha}-\lambda y^{\prime}(t)+\lambda \int_{0}^{t}(t-s)^{-\alpha} y^{\prime}(s) d s .
$$

Since $y^{\prime} \in \mathscr{C}([0, T])$, we conclude that, for this particular function, (cf. also Miller and Feldstein [11])

$$
\left\|u_{t t}\right\| \sim \lambda t^{-\alpha} \text { as } t \rightarrow 0
$$

\section{DisCRETIZATION IN SPACE}

In this section we shall derive the error estimate (1.5) stated in the introduction for the semidiscrete method (1.4).

For the analysis we introduce, following [1], the Ritz-Volterra projection $V_{h}$ defined for an appropriately smooth function $u$ by

$$
A\left(\left(V_{h} u-u\right)(t), \chi\right)=\int_{0}^{t} K(t-s) B\left(\left(V_{h} u-u\right)(s), \chi\right) d s, \quad \forall \chi \in S_{h}, t \geq 0 .
$$

We have the following error estimate:

Lemma 3. We have for the Ritz-Volterra projection

$$
\begin{aligned}
& \left\|\left(V_{h} u-u\right)(t)\right\|+h\left\|\left(V_{h} u-u\right)(t)\right\|_{1} \\
& \leq C h^{2} \sup _{s \leq t}\|u(s)\|_{2} \leq C h^{2}\left\{\left\|u_{0}\right\|_{2}+\int_{0}^{t}\left\|u_{t}\right\|_{2} d s\right\} .
\end{aligned}
$$

Proof. Let $W=V_{h} u$ and $\rho=W-u$. We begin with an $H^{1}$ estimate, and introduce also the standard Ritz projection $R_{h}$ defined by

$$
A\left(R_{h} u-u, \chi\right)=0, \quad \forall \chi \in S_{h} .
$$

We recall that (see Ciarlet [2, (18.3) and (19.13)]), under the assumption (1.3),

$$
\left\|R_{h} u-u\right\|+h\left\|R_{h} u-u\right\|_{1} \leq C h^{2}\|u\|_{2} \text {. }
$$

We have, using the definition of $W$, that, with $c>0$,

$$
\begin{aligned}
c\left\|\left(W-R_{h} u\right)(t)\right\|_{1}^{2} & \leq A\left(W-R_{h} u, W-R_{h} u\right)=A\left(\rho, W-R_{h} u\right)(t) \\
& =\int_{0}^{t} K(t-s) B\left(\rho(s),\left(W-R_{h} u\right)(t)\right) d s \\
& \leq C\left\|\left(W-R_{h} u\right)(t)\right\|_{1} \int_{0}^{t}(t-s)^{-\alpha}\|\rho(s)\|_{1} d s
\end{aligned}
$$


and hence

$$
\|\rho(t)\|_{1} \leq C \int_{0}^{t}(t-s)^{-\alpha}\|\rho(s)\|_{1} d s+\left\|\left(R_{h} u-u\right)(t)\right\|_{1} .
$$

Lemma 1 now implies

$$
\|\rho(t)\|_{1} \leq C_{T} \sup _{s \leq t}\left\|\left(R_{h} u-u\right)(s)\right\|_{1} \leq C_{T} h \sup _{s \leq t}\|u(s)\|_{2} .
$$

We next turn to the $L_{2}$ estimate, which will be derived by a duality argument, thus using

$$
\|\rho(t)\|=\sup _{\|\phi\|=1}(\rho(t), \phi) .
$$

For each such $\phi$, we let $\psi$ be the solution of

$$
A \psi=\phi \quad \text { in } \Omega, \quad \psi=0 \text { on } \partial \Omega,
$$

and recall that

$$
\|\psi\|_{2} \leq C\|\phi\|=C
$$

Then, for $\chi \in S_{h}$,

$$
(\rho(t), \phi)=A(\rho, \psi)=A(\rho, \psi-\chi)+A(\rho, \chi) .
$$

Here,

$$
\begin{aligned}
A(\rho, \chi) & =\int_{0}^{t} K(t-s) B(\rho(s), \chi) d s \\
& =\int_{0}^{t} K(t-s) B(\rho(s), \chi-\psi) d s+\int_{0}^{t} K(t-s)\left(\rho(s), B^{*} \psi\right) d s,
\end{aligned}
$$

and hence, with $\chi=R_{h} \psi$, using (3.2),

$$
\begin{aligned}
(\rho(t), \phi) & \leq C \sup _{s \leq t}\|\rho(s)\|_{1}\left\|R_{h} \psi-\psi\right\|_{1}+C \int_{0}^{t}(t-s)^{-\alpha}\|\rho(s)\| d s\|\psi\|_{2} \\
& \leq C\left\{h^{2} \sup _{s \leq t}\|u(s)\|_{2}+\int_{0}^{t}(t-s)^{-\alpha}\|\rho(s)\| d s\right\} .
\end{aligned}
$$

Thus,

$$
\|\rho(t)\| \leq C h^{2} \sup _{s \leq t}\|u(s)\|_{2}+C \int_{0}^{t}(t-s)^{-\alpha}\|\rho(s)\| d s,
$$

which by Lemma 1 completes the proof of Lemma 3.

We shall also need the following estimate for the time derivative of the error in the Ritz-Volterra projection.

Lemma 4. Under the assumptions of Lemma 3 we have, for $\rho=V_{h} u-u$,

$$
\int_{0}^{t}\left(\left\|\rho_{t}\right\|+h\left\|\rho_{t}\right\|_{1}\right) d s \leq C h^{2}\left\{\left\|u_{0}\right\|_{2}+\int_{0}^{t}\left\|u_{t}\right\|_{2} d s\right\} .
$$

Proof. Writing (3.1) in the form

$$
A(\rho(t), \chi)=\int_{0}^{t} K(s) B(\rho(t-s), \chi) d s, \quad \forall \chi \in S_{h},
$$


we obtain by differentiation

$$
A\left(\rho_{t}(t), \chi\right)=K(t) B(\rho(0), \chi)+\int_{0}^{t} K(s) B\left(\rho_{t}(t-s), \chi\right) d s .
$$

We begin with the $H^{1}$ estimate. We have, for $W=V_{h} u$,

$$
\begin{aligned}
c\left\|W_{t}-R_{h} u_{t}\right\|_{1}^{2} \leq & A\left(W_{t}-R_{h} u_{t}, W_{t}-R_{h} u_{t}\right) \\
= & A\left(\rho_{t}, W_{t}-R_{h} u_{t}\right)=K(t) B\left(\rho(0), W_{t}-R_{h} u_{t}\right) \\
& +\int_{0}^{t} K(t-s) B\left(\rho_{t}(s), W_{t}-R_{h} u_{t}\right) d s .
\end{aligned}
$$

Hence,

$$
\left\|\left(W_{t}-R_{h} u_{t}\right)(t)\right\|_{1} \leq C t^{-\alpha}\|\rho(0)\|_{1}+C \int_{0}^{t}(t-s)^{-\alpha}\left\|\rho_{t}(s)\right\|_{1} d s
$$

or

$$
\begin{aligned}
\left\|\rho_{t}(t)\right\|_{1} & \leq C t^{-\alpha}\|\rho(0)\|_{1}+\left\|\left(R_{h} u_{t}-u_{t}\right)(t)\right\|_{1}+C \int_{0}^{t}(t-s)^{-\alpha}\left\|\rho_{t}(s)\right\|_{1} d s \\
& \leq C h\left\{t^{-\alpha}\left\|u_{0}\right\|_{2}+\left\|u_{t}(t)\right\|_{2}\right\}+C \int_{0}^{t}(t-s)^{-\alpha}\left\|\rho_{t}(s)\right\|_{1} d s .
\end{aligned}
$$

Thus by Lemma 1,

$$
\left\|\rho_{t}(t)\right\|_{1} \leq C h\left\{t^{-\alpha}\left\|u_{0}\right\|_{2}+\left\|u_{t}(t)\right\|_{2}+\int_{0}^{t}(t-s)^{-\alpha}\left\|u_{t}(s)\right\|_{2} d s\right\},
$$

and finally

$$
\begin{aligned}
\int_{0}^{t}\left\|\rho_{t}\right\|_{1} d s & \leq C h\left\{\left\|u_{0}\right\|_{2}+\int_{0}^{t}\left\|u_{t}\right\|_{2} d s+\int_{0}^{t} \int_{0}^{s}(s-\tau)^{-\alpha}\left\|u_{t}(\tau)\right\|_{2} d \tau d s\right\} \\
& \leq C h\left\{\left\|u_{0}\right\|_{2}+\int_{0}^{t}\left\|u_{t}\right\|_{2} d s\right\} .
\end{aligned}
$$

We now turn to the $L_{2}$ bound and write, with the notation of Lemma 3 and using (3.3),

$$
\begin{aligned}
\left(\rho_{t}(t), \phi\right)= & A\left(\rho_{t}(t), \psi\right) \\
= & A\left(\rho_{t}(t), \psi-\chi\right)+\int_{0}^{t} K(t-s)\left[B\left(\rho_{t}(t), \psi-\chi\right)+\left(\rho_{t}(s), B^{*} \psi\right)\right] d s \\
& +K(t)\left[B(\rho(0), \psi-\chi)+\left(\rho(0), B^{*} \psi\right)\right] .
\end{aligned}
$$

With an appropriate choice of $\chi$ we obtain that

$$
\begin{aligned}
\left\|\rho_{t}(t)\right\| \leq & C h\left\{\left\|\rho_{t}(t)\right\|_{1}+\int_{0}^{t}(t-s)^{-\alpha}\left\|\rho_{t}(s)\right\|_{1} d s\right\}+C h^{2} t^{-\alpha}\left\|u_{0}\right\|_{2} \\
& +C \int_{0}^{t}(t-s)^{-\alpha}\left\|\rho_{t}(s)\right\| d s
\end{aligned}
$$

from which we conclude by Lemma 1 that

$$
\left\|\rho_{t}(t)\right\| \leq C h\left\{\left\|\rho_{t}(t)\right\|_{1}+\int_{0}^{t}(t-s)^{-\alpha}\left\|\rho_{t}(s)\right\|_{1} d s\right\}+C h^{2} t^{-\alpha}\left\|u_{0}\right\|_{2}
$$


After integration and using the $H^{1}$ estimate already derived we have

$$
\int_{0}^{t}\left\|\rho_{t}\right\| d s \leq C h \int_{0}^{t}\left\|\rho_{t}(s)\right\|_{1} d s+C h^{2}\left\|u_{0}\right\|_{2} \leq C h^{2}\left\{\left\|u_{0}\right\|_{2}+\int_{0}^{t}\left\|u_{t}(s)\right\|_{2} d s\right\},
$$

which thus completes the proof.

Theorem 2. Assume that $u_{0 h}$ is chosen so that

$$
\left\|u_{0 h}-u_{0}\right\| \leq C h^{2}\left\|u_{0}\right\|_{2} \text {. }
$$

Then for each $T>0$ there is a constant $C_{T}$ such that for the solutions of (1.1) and (1.4)

$$
\left\|u_{h}(t)-u(t)\right\| \leq C_{T} h^{2}\left\{\left\|u_{0}\right\|_{2}+\int_{0}^{t}\left\|u_{t}\right\|_{2} d s\right\} \quad \text { for } t \leq T
$$

Proof. In a standard fashion we write

$$
u_{h}-u=\left(u_{h}-V_{h} u\right)+\left(V_{h} u-u\right)=\theta+\rho .
$$

Lemma 3 immediately gives the desired estimate for $\rho$, so it remains to bound $\theta$.

We have directly from our definitions

$$
\left(\theta_{t}, \chi\right)+A(\theta, \chi)=\int_{0}^{t} K(t-s) B(\theta(s), \chi) d s+\left(\rho_{t}, \chi\right), \quad \forall \chi \in S_{h},
$$

and hence, setting $\chi=\theta$,

$$
\frac{1}{2} \frac{d}{d t}\|\theta\|^{2}+A(\theta, \theta) \leq C \int_{0}^{t}(t-s)^{-\alpha}\|\theta(s)\|_{1}\|\theta(t)\|_{1} d s+\left\|\rho_{t}\right\|\|\theta\| .
$$

By integration this yields

$$
\begin{aligned}
& \|\theta(T)\|^{2}+\int_{0}^{T}\|\theta\|_{1}^{2} d t \\
& \leq C\left\{\|\theta(0)\|^{2}+\int_{0}^{T} \int_{0}^{t}(t-s)^{-\alpha}\|\theta(s)\|_{1}\|\theta(t)\|_{1} d s d t+C \int_{0}^{T}\left\|\rho_{t}\right\|\|\theta\| d t\right\} .
\end{aligned}
$$

Using Lemma 2 with a suitable choice of $\varepsilon$ for the double integral, we thus have

$$
\begin{aligned}
\|\theta(T)\|^{2} & +\int_{0}^{T}\|\theta\|_{1}^{2} d t \\
& \leq C\left\{\|\theta(0)\|^{2}+\int_{0}^{T}\left\|\rho_{t}\right\|\|\theta\| d t+\int_{0}^{T}(T-t)^{-\alpha} \int_{0}^{t}\|\theta(s)\|_{1}^{2} d s d t\right\} .
\end{aligned}
$$

By Lemma 1, therefore, we obtain the bound

$$
\|\theta(T)\|^{2}+\int_{0}^{T}\|\theta\|_{1}^{2} d t \leq C_{T}\left\{\|\theta(0)\|^{2}+\int_{0}^{T}\left\|\rho_{t}\right\|\|\theta\| d t\right\},
$$

whence, using also Lemma 4 , and noting that $V_{h}(0)=R_{h}$,

$$
\begin{aligned}
\|\theta(T)\| & \leq C_{T}\left\{\|\theta(0)\|+\int_{0}^{T}\left\|\rho_{t}\right\| d t\right\} \\
& \leq C_{T}\left\{\left\|u_{0 h}-R_{h} u_{0}\right\|+h^{2}\left(\left\|u_{0}\right\|_{2}+\int_{0}^{T}\left\|u_{t}\right\|_{2} d s\right)\right\} .
\end{aligned}
$$


In view of our choice of $u_{h 0}$ this completes the proof of the desired estimate for $\theta$, and thus of the theorem.

\section{THE COMPLETELY DISCRETE SCHEME}

In this section we shall consider the completely discrete method (1.7).

In the next lemma we estimate a time-discrete $L_{1}\left(0, T ; L_{2}(\Omega)\right)$ type norm of the quadrature error

$$
\varepsilon_{n}(\phi)=\sum_{j=0}^{n-1} \kappa_{n-j} \phi\left(t_{j}\right)-\int_{0}^{t_{n}} K\left(t_{n}-s\right) \phi(s) d s,
$$

where $\kappa_{j}$ is defined by (1.6).

Lemma 5. For each $T>0$ there is a constant $C_{T}$ such that, if $\phi_{t} \in L_{1}\left(0, T ; L_{2}\right)$, then

$$
k \sum_{n=1}^{N}\left\|\varepsilon_{n}(\phi)\right\| \leq C_{T} k \int_{0}^{t_{N}}\left\|\phi_{t}(s)\right\| d s \quad \text { for } N k \leq T .
$$

Proof. By the definition of the $\kappa_{j}$ we have

$$
\varepsilon_{n}(\phi)=\sum_{j=0}^{n-1} \int_{t_{j}}^{t_{j+1}} K\left(t_{n}-s\right)\left(\phi\left(t_{j}\right)-\phi(s)\right) d s,
$$

so that by (1.2), for each $x \in \Omega$,

$$
\begin{aligned}
\left|\varepsilon_{n}(\phi)\right| & \leq \sum_{j=0}^{n-1} \int_{t_{j}}^{t_{j+1}}\left|K\left(t_{n}-s\right)\right| \int_{t_{j}}^{t_{j+1}}\left|\phi_{t}(\sigma)\right| d \sigma d s \\
& \leq C \sum_{j=0}^{n-1} \mu_{\alpha, n-j} \int_{t_{j}}^{t_{j+1}}\left|\phi_{t}(\sigma)\right| d \sigma,
\end{aligned}
$$

where

$$
\mu_{\alpha, j}=\int_{t_{j-1}}^{t_{j}} s^{-\alpha} d s=(1-\alpha)^{-1}\left(t_{j}^{1-\alpha}-t_{j-1}^{1-\alpha}\right) .
$$

By integration in $x$ and use of Minkowski's inequality this yields

$$
\left\|\varepsilon_{n}(\phi)\right\| \leq C \sum_{j=0}^{n-1} \mu_{\alpha, n-j} \int_{t_{j}}^{t_{j+1}}\left\|\phi_{t}\right\| d s .
$$

Hence, by interchanging the orders of summation we find

$$
\sum_{n=1}^{N}\left\|\varepsilon_{n}(\phi)\right\| \leq C \sum_{j=0}^{N-1} \sum_{n=j+1}^{N} \mu_{\alpha, n-j} \int_{t_{j}}^{t_{j+1}}\left\|\phi_{t}\right\| d s \leq C_{T} \int_{0}^{T_{N}}\left\|\phi_{t}\right\| d s,
$$

since

$$
\sum_{n=j+1}^{N} \mu_{\alpha, n-j}=\int_{0}^{t_{N-j}} s^{-\alpha} d s \leq C_{T}=(1-\alpha)^{-1} T^{1-\alpha} .
$$

This completes the proof.

The following two lemmas are discrete analogues of Lemmas 1 and 2, and are proved similarly to these. 
Lemma 6. Let $\mu_{\alpha, j}$ be defined by (4.1) and assume that $y_{n} \geq 0$ and satisfies

$$
y_{n} \leq b_{n}+\beta \sum_{j=0}^{n-1} \mu_{\alpha, n-j} y_{j} \text { for } n \geq 0
$$

where $b_{n} \geq 0, \beta \geq 0$. Then for each $T>0$ there is a constant $C_{T}$ such that

$$
y_{n} \leq b_{n}+C_{T} \sum_{j=0}^{n-1} \mu_{\alpha, n-j} b_{j} \quad \text { for } n k \leq T .
$$

Lemma 7. Let $K \in L_{1}(0, T)$, and let $\kappa_{j}$ be defined by (1.6). Then for each $\varepsilon>0$ there is a constant $C_{\varepsilon}=C_{\varepsilon}\left(\|K\|_{L_{1}(0, T)}\right)$ such that

$$
\left|\sum_{n=1}^{N} \sum_{j=0}^{n-1} \kappa_{n-j} f_{j} f_{n}\right| \leq \varepsilon \sum_{n=1}^{N} f_{n}^{2}+C_{\varepsilon} \sum_{n=0}^{N-1}\left|\kappa_{N-n}\right| \sum_{j=0}^{n-1} f_{j}^{2}
$$

The following error estimate is our main result of this section. Its proof will require the inverse estimate

$$
\|\chi\|_{1} \leq C h^{-1}\|\chi\|, \quad \forall \chi \in S_{h} .
$$

Theorem 3. Assume that $S_{h}$ satisfies (4.2) and that $u_{0 h}$ is chosen so that

$$
\left\|u_{0 h}-u_{0}\right\| \leq h^{2}\left\|u_{0}\right\|_{2} \text {. }
$$

Then for each $T>0$ there is a constant $C_{T}$ such that for the solutions of (1.7) and (1.1)

$$
\left\|U^{n}-u\left(t_{n}\right)\right\| \leq C_{T}\left(h^{2}+k\right)\left\{\left\|u_{0}\right\|_{2}+\int_{0}^{t_{n}}\left(\left\|u_{t t}\right\|+\left\|u_{t}\right\|_{2}\right) d s\right\} \quad \text { for } t \leq T .
$$

Proof. With $V_{h}$ the Ritz-Volterra projection introduced in (3.1), we write

$$
U^{n}-u\left(t_{n}\right)=\left(U^{n}-V_{h} u\left(t_{n}\right)\right)+\left(V_{h} u\left(t_{n}\right)-u\left(t_{n}\right)\right)=\theta^{n}+\rho^{n} .
$$

The term $\rho^{n}$ is estimated as desired by Lemma 3 . For $\theta^{n}$ we have by our definitions

$$
\left(\bar{\partial}_{t} \theta^{n}, \chi\right)+A\left(\theta^{n}, \chi\right)=\sum_{j=0}^{n-1} \kappa_{n-j} B\left(\theta^{j}, \chi\right)+\left(\tau_{n}, \chi\right)
$$

where

$$
\left(\tau_{n}, \chi\right)=\left(u_{t}^{n}-\bar{\partial}_{t} V_{h} u^{n}, \chi\right)+\sum_{j=0}^{n-1} \kappa_{n-j} B\left(V_{h} u^{j}, \chi\right)-\int_{0}^{t_{n}} K\left(t_{n}-s\right) B\left(V_{h} u(s), \chi\right) d s .
$$

Defining $B_{h}: H_{0}^{1} \rightarrow S_{h}$ by

$$
\left(B_{h} \phi, \chi\right)=B(\phi, \chi), \quad \forall \chi \in S_{h},
$$

we may write

$$
\tau_{n}=u_{t}^{n}-\bar{\partial}_{t} V_{h} u^{n}+\varepsilon_{n}\left(B_{h} V_{h} u\right)
$$

We shall show by an energy argument that

$$
\left\|\theta^{N}\right\| \leq C_{T}\left(\left\|\theta^{0}\right\|+k \sum_{n=1}^{N}\left\|\tau_{n}\right\|\right) \quad \text { for } N k \leq T .
$$


Assuming this for a moment, we then write $\tau_{n}=\sum_{l=1}^{4} \tau_{n}^{l}$, where

$$
\begin{aligned}
\tau_{n}^{1} & =u_{t}^{n}-\bar{\partial}_{t} u^{n}, \\
\tau_{n}^{2} & =\bar{\partial}_{t}\left(u^{n}-V_{h} u^{n}\right)=-\bar{\partial}_{t} \rho^{n}, \\
\tau_{n}^{3} & =\varepsilon_{n}\left(B_{h} u\right) \\
\tau_{n}^{4} & =\varepsilon_{n}\left(B_{h} \rho\right) .
\end{aligned}
$$

We have at once

$$
k \sum_{n=1}^{N}\left\|\tau_{n}^{1}\right\| \leq C k \sum_{n=1}^{N} \int_{t_{n-1}}^{t_{n}}\left\|u_{t t}\right\| d s=C k \int_{0}^{t_{N}}\left\|u_{t t}\right\| d s
$$

and, by Lemma 4 ,

$$
\begin{aligned}
k \sum_{n=1}^{N}\left\|\tau_{n}^{2}\right\| & \leq \sum_{n=1}^{N} \int_{t_{n-1}}^{t_{n}}\left\|\rho_{t}\right\| d s=\int_{0}^{t_{N}}\left\|\rho_{t}\right\| d s \\
& \leq C h^{2}\left\{\left\|u_{0}\right\|_{2}+\int_{0}^{t_{N}}\left\|u_{t}\right\|_{2} d s\right\} .
\end{aligned}
$$

To estimate $\tau_{n}^{3}$, we note that when $u$ is smooth, $B_{h} u=P_{h} B u$ and hence, by Lemma 5 ,

$$
k \sum_{n=1}^{N}\left\|\tau_{n}^{3}\right\| \leq C k \int_{0}^{t_{N}}\left\|P_{h} B u_{t}\right\| d s \leq C k \int_{0}^{t_{N}}\left\|u_{t}\right\|_{2} d s .
$$

Using the inverse assumption (4.2), we have

$$
\left(B_{h} \rho, \chi\right)=B(\rho, \chi) \leq C\|\rho\|_{1}\|\chi\|_{1} \leq C h^{-1}\|\rho\|_{1}\|\chi\|
$$

so that

$$
\left\|B_{h} \rho\right\| \leq C h^{-1}\|\rho\|_{1} .
$$

Hence, for $\tau_{n}^{4}$, we have by Lemmas 5 and 4 ,

$$
\begin{aligned}
k \sum_{n=1}^{N}\left\|\tau_{n}^{4}\right\| & \leq C_{T} k \int_{0}^{t_{N}}\left\|B_{h} \rho_{t}\right\| d s \\
& \leq C_{T} k h^{-1} \int_{0}^{t_{N}}\left\|\rho_{t}\right\|_{1} d s \leq C_{T} k\left\{\left\|u_{0}\right\|_{2}+\int_{0}^{t_{N}}\left\|u_{t}\right\|_{2} d s\right\} .
\end{aligned}
$$

Inserted into (4.5), these estimates show

$$
\left\|\theta^{N}\right\| \leq C_{T}\left\|u_{0 h}-R_{h} u_{0}\right\|+C_{T}\left(h^{2}+k\right)\left\{\left\|u_{0}\right\|_{2}+\int_{0}^{t_{N}}\left(\left\|u_{t t}\right\|+\left\|u_{t}\right\|_{2}\right) d s\right\} .
$$

In view of (4.3) this completes the proof.

It remains to show (4.5). For this we choose $\chi=\theta^{n}$ in (4.4), which yields

$$
\frac{1}{2} \bar{\partial}_{t}\left\|\theta^{n}\right\|^{2}+\frac{1}{2} k\left\|\bar{\partial}_{t} \theta^{n}\right\|^{2}+A\left(\theta^{n}, \theta^{n}\right)=\sum_{j=0}^{n-1} \kappa_{n-j} B\left(\theta^{j}, \theta^{n}\right)+\left(\tau_{n}, \theta^{n}\right),
$$

whence

$$
\bar{\partial}_{t}\left\|\theta^{n}\right\|^{2}+\left\|\theta^{n}\right\|_{1}^{2} \leq C \sum_{j=0}^{n-1} \mu_{\alpha, n-j}\left\|\theta^{j}\right\|_{1}\left\|\theta^{n}\right\|_{1}+C\left\|\tau_{n}\right\|\left\|\theta^{n}\right\|
$$


and, after summation,

$$
\begin{aligned}
\left\|\theta^{N}\right\|^{2}+k \sum_{n=1}^{N}\left\|\theta^{n}\right\|_{1}^{2} \leq & \left\|\theta^{0}\right\|^{2}+C k \sum_{n=1}^{N} \sum_{j=0}^{n-1} \mu_{\alpha, n-j}\left\|\theta^{j}\right\|_{1}\left\|\theta^{n}\right\|_{1} \\
& +C k \sum_{n=1}^{N}\left\|\tau_{n}\right\|\left\|\theta^{n}\right\| .
\end{aligned}
$$

Using Lemma 7 with $K(t)=C t^{-\alpha}$, we may conclude

$$
\begin{aligned}
\left\|\theta^{N}\right\|^{2}+k \sum_{n=1}^{N}\left\|\theta^{n}\right\|_{1}^{2} \leq & \left\|\theta^{0}\right\|^{2}+C k \sum_{n=1}^{N}\left\|\tau_{n}\right\|\left\|\theta^{n}\right\| \\
& +C \sum_{n=0}^{N-1} \mu_{\alpha, N-n}\left(k \sum_{j=0}^{n-1}\left\|\theta^{j}\right\|_{1}^{2}\right) .
\end{aligned}
$$

In combination with Lemma 6 , applied to $y_{N}=k \sum_{n=1}^{N}\left\|\theta^{j}\right\|_{1}^{2}$, this shows

$$
\left\|\theta^{N}\right\|^{2} \leq C_{T}\left(\left\|\theta^{0}\right\|^{2}+k \sum_{n=1}^{N}\left\|\tau_{n}\right\|\left\|\theta^{n}\right\|\right) \quad \text { for } N k \leq T,
$$

from which (4.5) follows.

This completes the proof.

\section{BIBLIOGRAPHY}

1. J. R. Cannon and Y. Lin, A priori $L^{2}$ error estimates for Galerkin method for nonlinear parabolic integro-differential equations., Manuscript, 1987.

2. P. G. Ciarlet, Basic error estimates for elliptic problems, Handbook of Numerical Analysis (P. G. Ciarlet and J. L. Lions, eds.), vol. II, North-Holland, Amsterdam, 1991, pp. 19-351.

3. A. Friedman and M. Shinbrot, Volterra integral equations in Banach space, Trans. Amer. Math. Soc. 126 (1967), 131-179.

4. R. C. Grimmer and A. J. Pritchard, Analytic resolvent operators for integral equations in Banach space, J. Differential Equations 50 (1983), 234-259.

5. M. L. Heard, An abstract parabolic Volterra integrodifferential equation, SIAM J. Math. Anal. 13 (1982), 81-105.

6. M.-N. LeRoux and V. Thomée, Numerical solution of semilinear integro-differential equations of parabolic type with nonsmooth data, SIAM J. Numer. Anal. 26 (1989), 1291-1309.

7. Y. Lin, V. Thomée, and L. Wahlbin, Ritz-Volterra projections to finite element spaces and applications to integro-differential and related equations, SIAM J. Numer. Anal. 28 (1991), 1047-1070.

8. P. Linz, Analytic and numerical methods for Volterra equations, SIAM Studies in Applied Mathematics, SIAM, Philadelphia, 1985.

9. A. Lorenzi and E. Sinestrari, An inverse problem in the theory of materials with memory, Nonlinear Anal. Theory Methods Appl. 12 (1988), 1317-1335.

10. A. Lunardi and E. Sinestrari, $C^{\infty}$-regularity of non-autonomous linear integro-differential equations of parabolic type, J. Differential Equations 63 (1986), 88-116.

11. R. K. Miller and A. Feldstein, Smoothness of solutions of Volterra integral equations with weakly singular kernels, SIAM J. Numer. Anal. 2 (1971), 242-258.

12. M. Renardy, W. J. Hrusa, and J. A. Nohel, Mathematical Problems in Viscoelasticity, Pitman Monographs and Surveys in Pure and Applied Mathematics, vol. 35, Longman Scientific and Technical, Harlow, Essex, 1987. 
13. I. H. Sloan and V. Thomée, Time discretization of an integro-differential equation of parabolic type, SIAM J. Numer. Anal. 23 (1986), 1052-1061.

14. V. Thomée and N.-Y. Zhang, Error estimates for semidiscrete finite element methods for parabolic integro-differential equations, Math. Comp. 53 (1989), 121-139.

15. E. G. Yanik and G. Fairweather, Finite element methods for parabolic and hyperbolic partial integro-differential equations, Nonlinear Anal. 12 (1988), 785-809.

Department of Mathematics, Xiangtan University, Xiangtan, People's Republic of CHINA

Department of Mathematics, Chalmers University of Technology, S-41262 Göteborg, SWEDEN

E-mail address: thomee@math.chalmers.se

Department of Mathematics, Cornell University, Ithaca, New York 14853

E-mail address: wahlbin@mssun7.msi.cornell.edu 